hypothesis is supported by the presence of IgA rheumatoid factor only in patients who have coeliac disease and IgA nephropathy and not in coeliac patients who do not have nephropathy.

The coexistence of these two specific disorders may explain why the proportion of patients in our population who had gliadin dependent mucosal lesions was definitely higher than that recently described by Hed $e t$ al in a large population of healthy blood donors screened for the presence of IgA-antigliadin antibodies. ${ }^{15}$ Both studies show that subclinical forms of coeliac disease are probably more common than was believed in the past and that in predisposed patients the renal disease may be its only clinical manifestation.

We thank Dr Paola Roggero for providing serum samples from the children with coeliac disease and Mrs Mascia Marchesini for typing the manuscript.

\section{References}

1 Hall RP, Stachura I, Cason J, Whiterside TL, Lawley TJ. IgA-containing circulating immune complexes in patients with IgA nephropathy. Am J Med 1983;74:56-63.

2 Woodroffe AJ, Gormly AA, McKenzie PE, et al. Immunologic studies in IgA nephropathy. Kidney Int 1980;18:366-74.
3 Coppo R, Basolo B, Martina G,et al. Circulating immune complexes containing IgA and IgM in patients with primary IgA nephropathy and with Henoch-Schönlein nephritis. Correlation with clinical and histologic signs of activity. Clin Nephrol 1982;18:230-9.

4 D'Amico G, Imbasciati E, di Belgioioso GB, et al. Idiopathic IgA mesangial nephropathy. Clinical and histological study of 374 patients. Medicine 1985;64:49-60.

D'Amico G. Idiopathic IgA mesangial nephropathy. Nephron 1985;41:1-13.

6 Amico G. Idiopachic 1 A mesangial nephropathy. Nephron 1985,41:1-13. idiopathic IgA mesangial nephropathy (Berger's disease). I Immunol 1986;137:536-41.

7 Czerkinsky C, Koopman WJ, Jackson S, et al. Circulating immune complexes and immunoglobulin A rheumatoid factor in patients with mesangial immunoglobulin A nephropathies. J Clin Invest 1986;7:1931-8.

8 Emancipator S, Gallo G, Lamm M. Experimental IgA nephropathy induced by oral immunization. $\mathcal{J}$ Exp Med 1983;157:572-82.

9 Moorthy AU, Zimmerman SW, Maxim PE. Dermatitis herpetiformis and celiac disease: association with glomerulonephritis, hypocomplementemia and circulating immune complexes. JAMA 1978;239:2019-20.

10 Helin H, Mustonen J, Reunala T, Pasternack A. IgA nephropathy associated coeliac disease and dermatitis herpetiformis. Arch Pathol Lab Med 1983;107:324-7.

11 Kendall MJ, Cox PS, Schneider R, Hawkins CF. Gluten substractions in coeliac disease. Lancet 1972;ii:1065-7.

12 Pereira AB, Theophilopulos AN, Dixon FJ. Detection and partial characterization of circulating immune complexes with solid-phase anti-C3. I I mmunol 1980;125:763-72.

13 Egido J, Blasco R, Sancho J, Lozano L, Crespo MS, Hernando L. Increased rates of polymeric IgA synthesis by circulating lymphoid cells in IgA mesangial glomerulonephritis. Clin Exp Immunol 1982;47:309-16.

14 Egido J, Blasco R, Sancho J, Lozano L. T cell dysfunction in IgA nephropathy: specific abnormalities in the regulation of IgA synthesis. Clin Immunol Immunopathol 1983;26:201-11. 15 Hed J, Lieden G, Ottosson E, et al. IgA anti-gliadin antibodies and jejunal mucosal lesions in healthy blood donors. Lancet 1986;ii:215.

(Accepted 2 April 1987)

\title{
Alcohol consumption and the risk of alcohol related cirrhosis in women
}

\author{
ROBYN NORTON, ROBERT BATEY, TERENCE DWYER, STEPHEN MACMAHON
}

\begin{abstract}
The risks in women of cirrhosis with a likely primary alcohol aetiology were estimated for various levels of alcohol consumption in a case-control study. Data were obtained from 41 women with a first diagnosis of cirrhosis who had no evidence of non-alcohol-related cirrhosis; three matched controls were interviewed for each case. Significant increases in the risk of cirrhosis were detected at levels of consumption between 41 and $60 \mathrm{~g}$ daily; above this level a dose-response relation was observed. The risk of cirrhosis did not appear to be influenced by other nutritional factors or history of liver disease or use of hepatotoxic drugs. One per cent of Australian women consume more than $40 \mathrm{~g}$ alcohol daily, yet more than $90 \%$ of women identified with cirrhosis consumed alcohol at this level.

Preventive interventions to reduce alcohol consumption in the small group of women who consume more than $40 \mathrm{~g}$ daily have the potential to reduce substantially the incidence of alcohol related cirrhosis.
\end{abstract}

\footnotetext{
School of Public Health and Tropical Medicine, University of Sydney, Australia

ROBYN NORTON, PHD, MPH, research fellow

TERENCE DWYER, MD, MPH, senior lecturer Drug and Alcohol Unit, Department of Medicine, Westmead Centre, Sydney,
Australia

ROBERT BATEY, FRACP, MRCP, director

Department of Medicine, The Prince Henry Hospital, Sydney, Australia STEPHEN MACMAHON, PHD, research fellow

Correspondence and requests for reprints to: Dr R Norton, Academic Department of Medicine, Royal Free Hospital, London NW3 2QG.
}

\section{Introduction}

There is concern about the potential consequences of increasing alcohol consumption in women. ${ }^{12}$ It has been suggested that women may develop liver damage at low levels of daily alcohol consumption. ${ }^{34}$ Few data are available, however, from which to determine the level of alcohol consumption at which an important increase in the risk of alcohol related cirrhosis occurs. Furthermore, we do not know whether other factors such as hepatotoxic drug use or a history of other liver disease interact with alcohol to increase the risk of cirrhosis. $^{56}$

We report the results of a case-control study which was designed to determine in women the relative risks of cirrhosis with a likely primary alcohol aetiology at various levels of daily alcohol consumption. The role of several factors hypothesised to interact with alcohol in the development of alcohol related cirrhosis was also investigated.

\section{Subjects and methods}

Over 20 months (July 1982 to February 1984) all women with a first diagnosis of cirrhosis of the liver admitted to eight main hospitals in the Sydney metropolitan region were identified prospectively. Women were eligible for selection as cases if they resided in the Sydney metropolitan area, were not lifetime abstainers from alcohol, and did not have cirrhosis with a primary aetiology known not to be related to alcohol. Fifty two women met these criteria. Of these, seven were considered too ill to be interviewed and $41(91 \%)$ of the remainder consented to participate. In 37 of these women the hospital diagnosis indicated cirrhosis of probable primary alcohol aetiology. We judged 31 women to have "definite" cirrhosis on the basis of a liver biopsy (in 10), an isotope liver scan (in 16), or, when liver biopsy was contraindicated, standard clinical signs and biochemical tests ${ }^{7}$ (in five). In six women there was evidence of "possible" cirrhosis; in these cases liver biopsy was not contraindicated and diagnoses were based on clinical signs and biochemical tests alone. The remaining four women had hospital diagnoses of cryptogenic cirrhosis. In two of these women diagnoses were 
based on biopsy specimens which were positive for cirrhosis but neither confirmed nor excluded alcohol as the primary aetiological factor; in the other two women the diagnosis was based on an isotope liver scan in one and clinical signs and biochemical tests in the other.

Controls were selected from women admitted to the medical and surgical wards of the same eight hospitals who were residents of the Sydney metropolitan area and who were not lifetime abstainers from alcohol. Because of concern that hospital populations may overrepresent heavy drinkers ${ }^{8}$ women admitted with conditions which might have been alcohol related, such as trauma, hypertension, or carcinoma of the head or neck, were not eligible for selection as controls. Three controls matched for hospital of admission and age (within five years) were selected for each case. Only $5 \%$ of the potential controls refused to participate in the study.

All cases and controls were interviewed in hospital by using a standardised questionnaire and standardised interviewing techniques. Demographic information and data on previous liver diseases, use of hepatotoxic drugs, daily nutritional intake, and family history of liver disease or alcohol related problems were sought in the questionnaire. Information on current and lifetime alcohol consumption was sought in an alcohol history section and the nutritional intake section of the questionnaire. The reliability of both methods of data collection on alcohol consumption has been shown..$^{39}$ In the alcohol history section data were collected on the usual number of days on which alcohol was consumed each month and the average number of drinks (each equivalent to $10 \mathrm{~g}$ alcohol) consumed per drinking day. After each interview information on alcohol consumption was sought from each subject's medical record. Information on alcohol consumption was also sought from the medical records of the potential cases and controls who were not interviewed. A measure of preadmission daily alcohol consumption was derived for each participant using the highest measure obtained in the alcohol history or nutritional intake section of the questionnaire or in the medical records. Data on this measure of alcohol consumption from three definite cases and eight controls were excluded from analyses because these women reported having altered their alcohol consumption for some time before admission as a consequence of their illness. An estimate of preadmission daily alcohol consumption could not be determined for one other control and two definite cases who declined to provide quantitative data on alcohol consumption.

Estimates of preadmission daily alcohol consumption were categorised as 0-20 g, 21-40 g, 41-60 g, 61-80 g, and over $80 \mathrm{~g}$. The planned analyses included calculating odds ratios (estimates of relative risk) and $95 \%$ confidence intervals for the risk of cirrhosis at levels of consumption above $20 \mathrm{~g}$ daily, using $0-20 \mathrm{~g}$ daily as the reference category. The risk of cirrhosis at each level of alcohol consumption was estimated in three separate analyses, one in the definite cases alone, another including both the definite and possible cases, and the third including all cases-definite, possible, and cryptogenic - in which a measure of preadmission daily alcohol consumption was available. Similar planned analyses entailed calculating odds ratios and $95 \%$ confidence intervals for the risk of cirrhosis at various levels of daily alcohol consumption adjusted for body weight using zero to $0.5 \mathrm{~g} / \mathrm{kg}$ body weight as the baseline. Multiple logistic regression analyses were conducted (BMDP statistical package) to determine whether any other variables of diet or drug or medical history contributed to the risks of developing cirrhosis after controlling for the preadmission daily alcohol consumption.

$\mathrm{p}$ Values for differences among groups were calculated by Student's $t$ test.

\section{Results}

The mean age in definite cases was 50 years (SD 10; range 27-71). This was similar to that in possible cases (mean 43; SD 15) but lower than that in cryptogenic cases (mean 68; SD 8). The mean preadmission daily alcohol consumption (g/day) in definite and possible cases was also similar and roughly $100 \mathrm{~g}$ greater than that in cryptogenic cases (table I). The mean preadmission consumption and mean consumption adjusted for body weight (g/day/kg) in both definite and possible cases were significantly greater than those in controls $(p<0.001)$. These measures of consumption in patients

TABLE I-Highest measure of preadmission daily alcohol consumption in cases and controls. Values are means (SEM in parentheses)

\begin{tabular}{|c|c|c|c|c|c|c|}
\hline \multirow{3}{*}{$\begin{array}{l}\text { Alcohol } \\
\text { consumption }\end{array}$} & \multicolumn{6}{|c|}{ Case groups } \\
\hline & \multicolumn{2}{|c|}{ Definite } & \multicolumn{2}{|c|}{ Possible } & \multicolumn{2}{|c|}{ Cryptogenic } \\
\hline & Cases & Controls & Cases & Controls & Cases & Controls \\
\hline $\begin{array}{l}\text { g/day } \\
\text { g/day } / \mathrm{kg}\end{array}$ & $\begin{array}{c}118(10 \cdot 2) \\
2 \cdot 1(0 \cdot 2)\end{array}$ & $\begin{array}{l}6 \cdot(1 \cdot 3) \\
0 \cdot 1(0 \cdot 02)\end{array}$ & $\begin{array}{r}112(19 \cdot 2) \\
2 \cdot 1(0 \cdot 2)\end{array}$ & $\begin{array}{l}8(2 \cdot 4) \\
0 \cdot 1(0 \cdot 05)\end{array}$ & $\begin{array}{c}12(12 \cdot 0) \\
0 \cdot 2(0 \cdot 2)\end{array}$ & $\begin{array}{l}7(3 \cdot 2) \\
0 \cdot 1(0 \cdot 06)\end{array}$ \\
\hline
\end{tabular}

with cryptogenic disease, however, were not significantly different from those of controls $(p>0.05)$.

Data on lifetime consumption from the three patients with definite cirrhosis who reported having altered their alcohol consumption before admission showed that their premorbid daily alcohol consumption (over $80 \mathrm{~g}$ in all cases) was similar to the preadmission daily alcohol consumption in other definite cases. In the 11 potential cases in which interview was not carried out the mean preadmission alcohol consumption estimated from medical records was not different from that estimated from the medical records of the interviewed group $(p>0.05)$. Mean preadmission daily alcohol consumption estimated from the medical records of the six potential controls who were not interviewed was not different from that estimated from the medical records of interviewed controls $(p>0.05)$.

The average frequency of alcohol consumption was greater in definite and possible cases ( 30 days a month) than in controls (six days a month) $(\mathrm{p}<0.01)$. Mean total lifetime consumption was also greater in definite cases $(755 \mathrm{~kg})$ than controls $(29 \mathrm{~kg})(\mathrm{p}<0.005)$; a similar difference between possible cases $(689 \mathrm{~kg})$ and controls $(27 \mathrm{~kg}$ ) did not achieve statistical significance $(\mathrm{p}>0.05)$. The average frequency of consumption and mean total lifetime consumption in cryptogenic cases (eight days a month and 39 $\mathrm{kg}$, respectively) and controls (nine days a month and $64 \mathrm{~kg}$ ) were similar $(p>0.05)$. In none of the groups was there a difference between cases and controls in the number of years of alcohol consumption $(p>0.05)$; the means for definite, possible, and cryptogenic cases were 25,15 , and 30 years, respectively.

Table II shows the distribution of cases and controls across the five categories of preadmission daily alcohol consumption. In roughly $80 \%$ of

TABLE II-Distribution of highest measure of preadmission consumption in cases and controls

\begin{tabular}{|c|c|c|c|c|c|c|}
\hline \multirow{3}{*}{$\begin{array}{l}\text { Alcohol } \\
\text { consumption } \\
\text { (g) }\end{array}$} & \multicolumn{6}{|c|}{ Case groups } \\
\hline & \multicolumn{2}{|c|}{ Definite } & \multicolumn{2}{|c|}{ Possible } & \multicolumn{2}{|c|}{ Cryptogenic } \\
\hline & Cases & Controls & Cases & Controls & Cases & Controls \\
\hline $0-20$ & 0 & 64 & 0 & 16 & 3 & 11 \\
\hline $21-40$ & 1 & 5 & 0 & 1 & 0 & 1 \\
\hline $41-60$ & 1 & 1 & 1 & 0 & 1 & 0 \\
\hline $61-80$ & 4 & 0 & 0 & 0 & 0 & 0 \\
\hline$>80$ & 20 & 0 & 5 & 0 & 0 & 0 \\
\hline
\end{tabular}

both definite and possible cases the women reported consuming more than $80 \mathrm{~g}$ daily. All women in the cryptogenic group consumed less than this, three of the four patients consuming $20 \mathrm{~g}$ or less daily. As none of the matched sets of cases and controls were concordant for category of alcohol consumption the planned matched analyses could not be conducted. In addition, as none of the patients in the definite or possible group consumed $20 \mathrm{~g}$ or less daily we could not calculate unmatched odds ratios using the $0-20$ g level as the reference category. Exact $95 \%$ confidence intervals, however, could be calculated from the unmatched data using a statistical program similar to that developed by Baptista and Pike. ${ }^{10}$ This allowed determination of the minimum preadmission daily alcohol consumption at which the risk of cirrhosis increased significantly above that at levels of daily consumption of $20 \mathrm{~g}$ or less. All analyses suggested typical dose response increases in the risk of cirrhosis (table III). Data from definite cases and controls indicated a highly significant increase in risk at levels of preadmission consumption between 61 and $80 \mathrm{~g}$ daily. Data from the definite and possible cases combined indicated a highly significant increase in risk at the 41 to $60 \mathrm{~g}$ level. The increase in risk at this level was also evident when cryptogenic cases were included in analyses with definite and possible cases. In data from definite cases and controls a significant increase in risk was evident at levels of preadmission daily alcohol consumption of between 0.5 and $1.0 \mathrm{~g} / \mathrm{kg}$ body

TABLE III-Exact $95 \%$ confidence intervals for relative risk of cirrhosis at various levels of preadmission alcohol consumption

\begin{tabular}{lrcc}
\hline & & \multicolumn{3}{c}{ Case groups } \\
\cline { 2 - 4 } $\begin{array}{l}\text { Alcohol } \\
\text { consumption (g) }\end{array}$ & Definite & $\begin{array}{c}\text { Definite and } \\
\text { possible }\end{array}$ & $\begin{array}{c}\text { Definite, possible, } \\
\text { and cryptogenic }\end{array}$ \\
\hline $0-20$ & - & - & - \\
$21-40$ & 0.27 to $\infty$ & $0 \cdot 29$ to $\infty$ & 0.07 to $61 \cdot 52$ \\
$41-60$ & 0.82 to $\infty$ & 6.32 to $\infty$ & 4.70 to 4818.01 \\
$61-80$ & 25.87 to $\infty$ & $32 \cdot 41$ to $\infty$ & 17.30 to $\infty$ \\
$>80$ & 162.64 to $\infty$ & 257.54 to $\infty$ & 139.93 to $\infty$ \\
\hline
\end{tabular}


weight ( $95 \%$ confidence interval $7 \cdot 47$ to $\infty$ ) in comparison with levels below $0.5 \mathrm{~g} / \mathrm{kg}$. An increase in risk at this level was also evident when possible and cryptogenic cases were included in the analyses.

Of all the non-alcohol variables investigated, only mean daily protein intake differed significantly between cases and controls. Univariate analyses suggested a $35 \%$ lower mean protein intake in definite and possible cases (40 $\mathrm{g}$ daily) compared with controls $(61 \mathrm{~g}$ daily) $(\mathrm{p}<0.05)$. In multiple logistic regression analyses, however, protein intake could not be shown to contribute significantly to the risk of cirrhosis after controlling for preadmission daily alcohol consumption $(p>0 \cdot 10)$.

\section{Discussion}

These results show that the risk of cirrhosis with a likely primary alcohol aetiology is significantly increased in women who consume between 41 and $60 \mathrm{~g}$ alcohol daily. The observed increase in risk at this level of preadmission daily alcohol consumption did not appear to be mediated by any of the other nutritional, drug use, or medical history factors studied. The increase in risk in those consuming 41 to $60 \mathrm{~g}$ daily was evident from analyses of data from the combined definite and possible cases; inclusion of data from the cryptogenic cases did not alter this result.

The similarity of data on alcohol consumption, age, and other factors in the definite and possible cases suggests that these cases constituted a homogenous group. By contrast, the cryptogenic cases were quite distinct from the other two case groups; patients were older and their mean preadmission daily alcohol consumption was substantially lower than that in either the definite or possible cases. A primary alcohol aetiology seems unlikely in three of the cryptogenic cases, in each of which the patient reported consuming less than $10 \mathrm{~g}$ alcohol a month. A primary alcohol aetiology is more likely, however, for the other cryptogenic case, in which the patient reported consuming $54 \mathrm{~g}$ alcohol daily; similar levels of alcohol consumption were reported in one definite and one possible case.

A preadmission daily alcohol consumption less than $41 \mathrm{~g}$ was reported in one definite case. This woman had a biopsy diagnosis of alcohol related cirrhosis and her alcohol consumption was consistently reported as $30 \mathrm{~g}$ daily in each source of data about alcohol intake. This level of consumption was confirmed by her spouse. This woman was, however, of low body weight and her preadmission consumption exceeded $0.5 \mathrm{~g} / \mathrm{kg}$. Levels of consumption between 0.5 and $1.0 \mathrm{~g} / \mathrm{kg}$ were associated with a significantly increased risk of cirrhosis. Interestingly, in all definite and possible cases the patients consumed more than $0.5 \mathrm{~g}$ alcohol $/ \mathrm{kg}$ daily, whereas only $3 \%$ of controls consumed alcohol at this level.

Assessment of the validity of the study findings requires consideration of several potential sources of bias. In any study which measures self reported alcohol intake the possibility of underestimation of alcohol consumption cannot be excluded. ${ }^{11}$ In this study the highest of the three measurements of alcohol consumption was used in the risk analyses. Nevertheless, if this measure still represents an underestimate of true consumption then the minimum level of consumption at which the risk of cirrhosis is increased significantly may be greater than 41-60 $\mathrm{g}$ daily. The potential bias introduced by the possible inclusion of women without cirrhosis or women with cirrhosis of non-alcohol aetiology among those cases without a biopsy diagnosis was also considered. The mean preadmission daily alcohol consumption of possible cases and definite cases diagnosed other than by biopsy was not different from that of cases diagnosed by biopsy. In addition, women in five of the six definite cases who consumed $80 \mathrm{~g}$ or less daily were diagnosed by biopsy. Thus our results are unlikely to be biased by the inclusion of data from cases without a biopsy diagnosis. The estimates of risk in the study are also unlikely to be biased by the selection of either cases or controls. The premorbid consumption by at least three of the five women with definite cirrhosis who did not contribute data on preadmission consumption was similar to the preadmission consumption in other definite cases. The mean daily consumption in potential cases and controls who were not interviewed was not different from that in study cases and controls. In addition, the frequency and quantity of alcohol consumption in study controls was almost identical with that reported for women of similar age in a random sample of women in the Australian urban population. ${ }^{12}$

In summary, these results indicate significant increases in the risk of alcohol related cirrhosis at levels of consumption greater than $40 \mathrm{~g}$ daily. Previous studies have reported an increased risk of ascitic cirrhosis of unspecified primary aetiology at levels of consumption between 21 and $40 \mathrm{~g}$ daily in both men and women. ${ }^{3413} \mathrm{We}$ do not know, however, whether this represents a true increase in the risk of cirrhosis with a primary alcohol aetiology or a contributory role of alcohol in the development of cirrhosis of other primary aetiology. In this study the reported consumption of $30 \mathrm{~g}$ daily in one case suggests that alcohol related cirrhosis may develop at levels of consumption between 21 and $40 \mathrm{~g}$ daily, but it is unclear whether the risk at this level is different from that at levels below $20 \mathrm{~g}$ daily. Nevertheless the absolute risk of developing alcohol related cirrhosis at levels of consumption between 21 and $40 \mathrm{~g}$ is undoubtedly slight given both the low incidence of cirrhosis in Australian women ${ }^{14}$ and the finding that $7 \%$ of controls in the study consumed alcohol at this level. At levels of alcohol consumption above $40 \mathrm{~g}$ daily the absolute risk increased sharply. In our study more than $90 \%$ of women in identified cases consumed alcohol above this level. Only $1 \%$ of controls, however, reported consumption of more than $40 \mathrm{~g}$ daily, which is a similar proportion to that in women in the general population. ${ }^{12}$

In terms of both individual and population attributable risks preventive interventions to reduce consumption of alcohol ${ }^{15}$ in the small group of women who consume more than $40 \mathrm{~g}$ daily have the greatest potential to produce important reductions in the incidence of alcohol related cirrhosis. Preventive interventions at lower levels of consumption might also be beneficial, particularly if postulated genetic factors can be shown to identify women who are most susceptible to alcohol induced liver damage. ${ }^{16}$

We thank Mr Philip Mock and Professor Geoffrey Berry (Department of Public Health, University of Sydney) for their advice and help with the statistical analyses. This study was supported by a grant from the New South Wales Drug and Alcohol Authority.

\section{References}

1 Anonymous. Women and alcohol. Lancet 1986;ii:353.

2 Blume SB. Women and alcohol. A review. $7 A M A$ 1986;256:1467-70.

3 Pequignot G, Tuyns AJ. Rations d'alcool consommees "declarees" et risques pathologiques. In: Symposium Franco-Britanique sur l'alcoolisme. INSERM/MRC. 27-28 Novembre 1975. Vol 54. Paris: INSERM, 1975:23.

4 Tuyns AJ, Pequignot G. Greater risk of ascitic cirrhosis in females in relation to alcohol consumption. Int f Epidemiol 1984;13:53-7.

5 Norton R, Batey R. Why do women develop alcoholic liver disease more readily than men? Australian AlcoholiDrug Review 1983;2:48-52.

6 Saunders JB, Wodak A, Williams R. What determines susceptibility to liver damage from alcohol?: a discussion paper. $\mathcal{F} R$ Soc Med 1984;77:204-16.

7 Leiber CS. Medical disorders of alcoholism. Philadelphia: W B Saunders, 1982:295.

8 Williams AT, Harding Burns F, Morey S. Prevalence of alcoholism in a Sydney teaching hospital: some aspects. Med $\mathcal{Y}$ Aust 1978;ii:608-11.

9. Skinner HA, Sheu WJ. Reliability of alcohol use indices: the lifetime drinking history and the MAST. F Stud Alcohol 1982;43:1157-70.

10 Baptista J, Pike MC. Exact two-sided confidence limits for the odds ratios in $2 \times 2$ tables. Applied Statistics 1977;26:214-20.

11 Pernanen K. Validity of survey data on alcohol use. In: Gibbins RJ, Israel Y, Kalant H, Popham RE, Schmidt W, Smart RG, eds. Research advances in alcohol and drug problems. Vol 1. New York: Wiley \& Sons, 1974:335.

12 National Heart Foundation of Australia. Risk factor prevalence study, No 1. 1980. Canberra: National Heart Foundation, 1981 .

13 Pequignot G, Tuyns AJ, Berta JL. Ascitic cirrhosis in relation to alcohol consumption. Int 7 Epidemiol 1978;7:113-20.

14 Norton R. Cirrhosis mortality and morbidity in Australian women. Sydney: New South Wales Drug and Alcohol Authority, 1986. (Research Grant Report Series B 86/2).

15 Babor TF, Ritson EB, Hodgson RJ. Alcohol-related problems in the primary health care setting: a review of early intervention strategies. $B r \mathcal{F}$ Addict 1986;81:23-46.

16 Johnson R, Williams R. Genetic and environmental factors in the individual susceptibility to the development of alcoholic liver disease. Alcohol and Alcoholism 1985;20:137-60.

(Accepted 1 May 1987) 